\title{
Adaptation to different mouth shapes influences visual perception of ambiguous lip speech
}

\author{
Benedict C. Jones \\ University of Aberdeen, Aberdeen, Scotland \\ DAVID R. FeINBERG \\ McMaster University, Hamilton, Ontario, Canada \\ Patricia E. G. Bestelmeyer \\ University of Glasgow, Glasgow, Scotland \\ LiSA M. DeBrUINE \\ University of Aberdeen, Aberdeen, Scotland \\ AND \\ Anthony C. LitTle \\ University of Stirling, Stirling, Scotland
}

\begin{abstract}
We investigated the effects of adaptation to mouth shapes associated with different spoken sounds (sustained $/ \mathrm{m} /$ or $/ \mathrm{u} /$ ) on visual perception of lip speech. Participants were significantly more likely to label ambiguous faces on an $/ \mathrm{m} /$-to-/u/ continuum as saying / $\mathrm{u}$ / following adaptation to $/ \mathrm{m} /$ mouth shapes than they were in a preadaptation test. By contrast, participants were significantly less likely to label the ambiguous faces as saying $/ \mathrm{u} /$ following adaptation to $/ \mathrm{u} /$ mouth shapes than they were in a preadaptation test. The magnitude of these aftereffects was equivalent when the same individual was shown in the adaptation and test phases of the experiment and when different individuals were presented in the adaptation and test phases. These findings present novel evidence that adaptation to natural variations in facial appearance influences face perception, and they extend previous research on face aftereffects to visual perception of lip speech.
\end{abstract}

Many studies have used visual adaptation to investigate whether recent visual experience influences face perception (e.g., Calder, Jenkins, Cassel, \& Clifford, 2008; DeBruine, Jones, Unger, Little, \& Feinberg, 2007; Little, DeBruine, \& Jones, 2005; Rhodes, Jeffery, Watson, Clifford, \& Nakayama, 2003; Webster, Kaping, Mizokami, \& Duhamel, 2004; Webster \& MacLin, 1999). For example, adaptation to faces with expanded feature spacing causes the feature spacing of unmanipulated faces to appear contracted, whereas adaptation to faces with contracted feature spacing causes the feature spacing of unmanipulated faces to appear expanded (Webster \& MacLin, 1999). Other studies have shown that adaptation to faces with either expanded or contracted feature spacing causes the adapted feature spacing to appear more normal and more attractive (Rhodes et al., 2003). This effect of adaptation on attractiveness judgments is thought to reflect the correlation between perceived normality and attractiveness (DeBruine et al., 2007; Rhodes et al., 2003).

The effects of visual adaptation on face perception occur when the same individuals (see, e.g., Yamashita, Hardy,
De Valois, \& Webster, 2005) and when different individuals (see, e.g., Rhodes et al., 2003; Yamashita et al., 2005) are shown at adaptation and test. Furthermore, aftereffects for normality and attractiveness judgments of faces occur when the faces shown during the adaptation and test phases differ in size (e.g., Bestelmeyer et al., 2008; Jones, DeBruine, \& Little, 2008; Little et al., 2005) or in orientation (e.g., Jeffery, Rhodes, \& Busey, 2006; Rhodes et al., 2003), suggesting that face aftereffects reflect adaptation of neurons that code high-level aspects of faces, rather than low-level (e.g., retinal) aftereffects (Bestelmeyer et al., 2008; Jeffery et al., 2006; Leopold, O'Toole, Vetter, \& Blanz, 2001; Little et al., 2005; Rhodes et al., 2003). Consistent with this proposal, neurobiological evidence shows that adaptation to faces alters responses in the lateral fusiform gyrus and in the superior temporal sulcus (e.g., Winston, Henson, Fine-Goulden, \& Dolan, 2004) brain regions that appear to be involved in the processing of high-level aspects of faces rather than the coding of low-level physical differences among different face patterns (Haxby, Hoffman, \& Gobbini, 2000; Rotshtein, Hen-

B. C. Jones, ben.jones@abdn.ac.uk 
son, Treves, Driver, \& Dolan, 2005). That aftereffects can be simultaneously induced in opposite directions for faces from different social categories also implicates adaptation of mechanisms that are sensitive to high-level aspects of faces (see, e.g., Bestelmeyer et al., 2008; Jaquet, Rhodes, \& Hayward, 2007; Little et al., 2005, Little, DeBruine, Jones, \& Waitt, 2008).

Although most early behavioral studies of the effects of visual adaptation to facial cues used grossly distorted images as the adapting stimuli (e.g., Rhodes et al., 2003; Webster \& MacLin, 1999), other studies have emphasized the importance of demonstrating aftereffects for adaptation to more natural variations in appearance (e.g., Webster et al., 2004). For example, studies have demonstrated effects of adaptation to facial identity (e.g., Leopold et al., 2001), sex (e.g., Webster et al., 2004), ethnicity (e.g., Webster et al., 2004), gaze direction (Calder et al., 2008; Jenkins, Beaver, \& Calder, 2006), emotional expression (e.g., Bestelmeyer et al., 2010; Hsu \& Young, 2004; Webster et al., 2004), and head orientation (e.g., Fang, Ijichi, $\& \mathrm{He}, 2007)$, whereby adaptation decreases sensitivity to the adapted characteristic in more ambiguous stimuli. For example, adaptation to a particular expression decreases the likelihood of ambiguous expressions being labeled as showing the adapted emotion (Bestelmeyer et al., 2010; Webster et al., 2004). Similarly, adaptation to faces with left-averted gaze causes faces with subtle left-deviated gaze to be perceived as averting their gaze to the right (Calder et al., 2008; Jenkins et al., 2006).

Lip-read speech plays an important role in social interactions, as was demonstrated by the McGurk effect (McGurk \& MacDonald, 1976), in which the spoken syllable /ba/ presented in combination with video of a person displaying the mouth shapes associated with the syllable /ga/ induced perceptions of the syllable /da/ in the majority of participants. Subsequent studies have demonstrated that dubbing ambiguous sounds onto video footage of a person articulating other sounds can bias perceptions of these sounds on subsequent trials, suggesting that aftereffects can be induced for perceptions of auditory speech following adaptation to ambiguous speech in the auditory domain (Bertelson, Vroomen, \& de Gelder, 2003). Although this finding shows that adaptation to ambiguous sounds can bias subsequent perception of speech, we know of no research demonstrating that visual adaptation to mouth shapes associated with certain spoken sounds influences visual perception of lip speech. Such effects would present novel evidence that adaptation to natural variations in appearance affects face perception and would extend previous findings for face aftereffects to include perceptions of lip speech. As mentioned previously, adaptation to facial cues appears to decrease sensitivity to the adapted characteristics in ambiguous stimuli (see, e.g., Bestelmeyer et al., 2010; Webster et al., 2004), suggesting that, following adaptation to mouth shapes associated with a given spoken sound, participants would be less likely to label ambiguous lip speech as saying the sound associated with the adapting stimuli. Such a pattern of results would demonstrate that visual adaptation to mouth shapes associated with different spoken sounds influences perceptions of ambiguous lip speech. Xu, Dayan, Lipkin, and Qian (2008) recently demonstrated that adaptation to concave and convex lines was sufficient to induce aftereffects for perceptions of happiness and sadness when judging the emotional expression of faces, suggesting that adaptation to different mouth shapes can induce aftereffects, at least for perceptions of emotional expressions. However, some models of face perception would suggest that dissociable cognitive routes underpin perceptions of lip speech and emotional expressions (e.g., Bruce \& Young, 1986). Such models suggest that findings for effects of adaptation to different mouth shapes that were observed for emotion perception may not necessarily generalize to lip-speech perception.

Studies of auditory recalibration of lip-speech perception suggest that hearing spoken sounds can bias subsequent perception of lip speech by increasing the tendency for ambiguous lip speech to be perceived as saying the previously heard sound (Eisner \& McQueen, 2005; van Linden \& Vroomen, 2007; Vroomen \& Baart, 2009). Thus, although previous findings for face aftereffects suggest that viewing mouth shapes associated with a given spoken sound may decrease sensitivity to that mouth shape in ambiguous stimuli, studies of auditory recalibration of lip speech raise the possibility that viewing mouth shapes associated with a given spoken sound may increase the likelihood of participants subsequently perceiving ambiguous lip speech to be saying that sound. Of course, the mechanisms and processes that underpin auditory and visual recalibration of lip speech need not function in the same way. Thus, testing the nature of the effects of visual adaptation to different mouth shapes on perceptions of ambiguous lip speech presents an opportunity to test for evidence that the processes and mechanisms that underpin auditory and visual recalibration of lip-speech perception function in different ways. If visual adaptation to different mouth shapes biases perceptions of ambiguous lip speech in ways consistent with previous findings in the face aftereffects literature (i.e., decreasing sensitivity to the adapting characteristics), such results would suggest that the mechanisms and processes that contribute to auditory and visual recalibration of lip-speech perception function in different ways.

We tested whether visual adaptation to faces with mouth shapes associated with one of two common speech sounds (sustained $/ \mathrm{m} / \mathrm{and} / \mathrm{u} /$ ) biases perceptions of mouth shapes in which the sound that appears to be being spoken is ambiguous. In an initial preadaptation test, we first assessed participants' perceptions of faces from continua that morphed (in 15 steps) from an image of an individual saying $/ \mathrm{m} /$ (sustained) to an image of that individual saying $/ \mathrm{u} /$ (sustained). Next, we adapted participants to faces with mouth shapes associated with either the $/ \mathrm{m} /$ or $/ \mathrm{u} /$ sounds in an adaptation phase. Finally, in a postadaptation test, we retested participants' perceptions of the faces shown in the preadaptation test. To assess whether the effects of adaptation to mouth shapes associated with different sounds not only affect perceptions of the adapted identity but also generalize to perceptions of different (i.e., novel) identities, the identity shown in the adaptation phase was manipulated (same vs. different identity from that seen at test). 


\section{METHOD}

\section{Participants}

Four hundred twelve participants (263 female; mean age $=$ 25.33 years, $S D=10.44$ ) took part.

\section{Stimuli}

Stimuli were 16 face image continua, each continuum depicting a young white adult male saying $/ \mathrm{m} /$ morphing, in 15 equally spaced steps, to an image of the same male saying / $\mathrm{u}$ / (see Tiddeman, Perrett, \& Burt, 2001, for technical details of the computer-graphic morphing method used to generate these images). Images of different males were used for each of these 16 continua. Figure 1 shows examples of face images taken from two of these continua. Images were masked so that hairstyle and clothing were not visible.

\section{Procedure}

The experiment consisted of three parts: an initial preadaptation test, an adaptation phase, and a postadaptation test. In the preadaptation test, participants were shown the 15 images from one of the 16 continua in a fully randomized order and indicated whether the face was saying $/ \mathrm{m} /$ (i.e., "mmm") or /u/ (i.e., "ooo"). Each image remained on screen until the participant had made a response (mean response time $[\mathrm{RT}]=2,400 \mathrm{msec}, S D=930$ ). The next image was then presented immediately. In order to reduce possible effects of low-level (i.e., retinal) aftereffects, the images shown in the preand postadaptation tests were $30 \%$ larger than those shown in the adaptation phase, following the procedures of previous studies (e.g., Bestelmeyer et al., 2008; Bestelmeyer et al., 2010; DeBruine et al., 2007; Jones et al., 2008)

In the adaptation phase, participants viewed a slideshow of the three images closest to either the $/ \mathrm{m} /$ or the $/ \mathrm{u} /$ end of the continuum (i.e., the $/ \mathrm{m} /$ or $/ \mathrm{u} /$ adaptation condition, respectively). Each image was presented eight times for $2 \mathrm{sec}$ ( $48 \mathrm{sec}$ total). The order of presentation was fully randomized. Participants were instructed to watch the faces closely.

The postadaptation test immediately followed the adaptation phase and was identical to the preadaptation test. The mean RT in the postadaptation phase test was $1,890 \mathrm{msec}(S D=1,180)$.

Each participant was randomly allocated 1 of the 16 continua as test images and was randomly allocated to either the $/ \mathrm{m} /$ or $/ \mathrm{u} /$ adaptation condition. Half of the participants who were allocated a given continuum as test images were adapted using images of the same individual shown at test (same identity adaptation), and half were adapted using images of a different individual from the one shown at test (different identity adaptation).

The experiment was run online. Previous studies using Web-based methods have demonstrated face aftereffects that are consistent with those reported in laboratory studies (Bestelmeyer et al., 2010; DeBruine et al., 2007; Jones et al., 2008).

\section{RESULTS}

For responses in the preadaptation tests, we fitted a logistic curve to the data for each of the 16 continua separately for each combination of lip-speech adaptation condition $(/ \mathrm{m} /$ or $/ \mathrm{u} /)$ and adaptation identity (same vs. different identity as at test). Excellent fits were obtained for all conditions (mean $R^{2}$ across conditions $=.99$ ).

Next, we calculated the point of subjective equality (PSE; García-Pérez \& Alcalá-Quintana, 2005) separately for each condition and for each of the continua. PSE values refer to the point on the test continuum where the face was equally likely to be labeled as saying $/ \mathrm{m} /$ or $/ \mathrm{u} /$ and can range from 1 (veridical $/ \mathrm{m} /$ image perceived as ambiguous) to 15 (veridical /u/ image perceived as ambiguous). Higher values indicate that the PSE was closer to the $/ \mathrm{u} /$ end of the continuum and lower values indicate that the PSE was closer to the $/ \mathrm{m} /$ end of the continuum. We also calculated corresponding PSEs, using data from the postadaptation phase tests.

PSEs were analyzed using a repeated measures ANOVA with the repeated measures of lip-speech adaptation $(/ \mathrm{m} /$ or $/ \mathrm{u} /$ ), adaptation identity (same or different identity as at test) and test phase (preadaptation or postadaptation). The individual test continua served as the unit of analysis. Descriptive statistics for each combination of factors in this analysis are shown in Table 1. The repeated measures ANOVA revealed a significant main effect of lip-speech adaptation $\left[F(1,15)=15.43, p<.001, \eta_{\mathrm{p}}^{2}=\right.$ .72], which was qualified by a significant interaction between lip-speech adaptation and test phase $[F(1,15)=$ $32.50, p<.001, \eta_{\mathrm{p}}^{2}=.68$; see Figure 2]. There were no other significant effects (all $F_{\mathrm{S}}<2.04$, all $p \mathrm{~s}>.17$, all $\left.\eta_{\mathrm{p}}^{2} \mathrm{~s}<.12\right)$.

Next, we used paired-samples $t$ tests to interpret the significant interaction between lip-speech adaptation and test phase. Since the three-way interaction among lip-speech adaptation, test phase, and adaptation identity was not significant $\left[F(1,15)=1.19, p=.29, \eta_{\mathrm{p}}^{2}=.07\right]$,
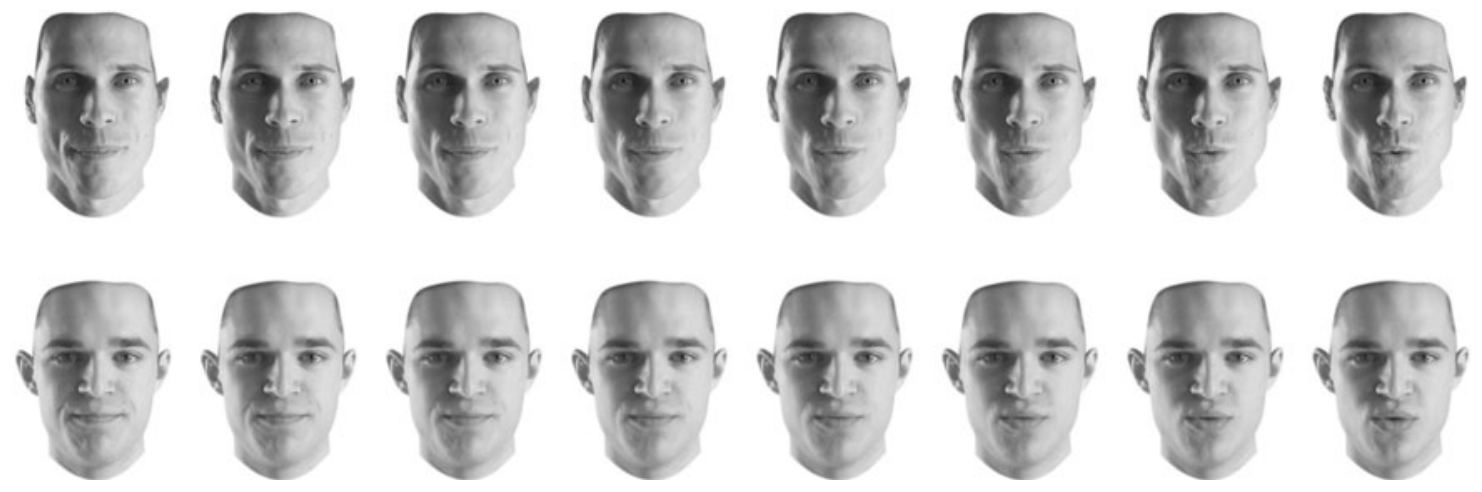

Figure 1. Examples from the 15-step morphed continua used to assess perceptions of lip speech in the pre- and postadaptation phase tests. The figure shows the 1st (leftmost image in each continuum), 3rd, 5th, 7th, 9th, 11th, 13th, and 15th (rightmost image in each continuum) steps from two continua. The leftmost image in each row is the veridical (i.e., $100 \%) / \mathrm{m} /$ image. The rightmost image in each row is the veridical $/ \mathrm{u} / \mathrm{image}$. 
Table 1

Descriptive Statistics for Each Combination of Lip-Speech Adaptation ( $/ \mathrm{m} /$ or $/ \mathrm{u} /$ ), Adaptation Identity (Same or Different Identity As at Test), and Test Phase (Pre- or Postadaptation Test) in Our Initial Analysis

\begin{tabular}{cclcc}
\hline $\begin{array}{c}\text { Lip-Speech } \\
\begin{array}{c}\text { Adaptation } \\
\text { Condition }\end{array}\end{array}$ & $\begin{array}{c}\text { Adaptation } \\
\text { Identity } \\
\text { Condition }\end{array}$ & Test Phase & $\begin{array}{c}\text { Mean Point } \\
\text { of Subjective } \\
\text { Equality }\end{array}$ & SEM \\
\hline /m/ & Different & Preadaptation & 7.37 & 0.29 \\
& & Postadaptation & 7.06 & 0.28 \\
& Same & Preadaptation & 7.69 & 0.30 \\
& & Postadaptation & 6.90 & 0.22 \\
$/ \mathrm{u} /$ & \multirow{2}{*}{ Different } & Preadaptation & 7.54 & 0.31 \\
& & Postadaptation & 8.32 & 0.30 \\
& \multirow{2}{*}{ Same } & Preadaptation & 7.53 & 0.21 \\
& & Postadaptation & 8.39 & 0.21 \\
\hline
\end{tabular}

Note-Individual test continua served as the unit of analysis.

data were collapsed across the adaptation identity factor. Planned comparisons showed that values for the PSE were increased following adaptation to $/ \mathrm{u} /$ mouth shapes $\left[t(15)=-4.62, p<.001, \eta_{\mathrm{p}}^{2}=.59\right]$ but were decreased following adaptation to $/ \mathrm{m} /$ mouth shapes $[t(15)=4.44$, $\left.p<.001, \eta_{\mathrm{p}}^{2}=.57\right]$. Additionally, PSEs did not differ significantly between participants in the $/ \mathrm{m} /$ and $/ \mathrm{u} / \mathrm{lip}$ speech adaptation conditions in the preadaptation phase test $\left[t(15)=0.07, p=.95, \eta_{\mathrm{p}}^{2}<.001\right]$ but did differ significantly in the postadaptation phase test $[t(15)=8.50$, $\left.p<.001, \eta_{\mathrm{p}}^{2}=.83\right]$.

Figures $3 \mathrm{~A}$ and $3 \mathrm{~B}$ show the logistic curves fitted to data from the pre- and postadaptation phase tests for participants adapted to $/ \mathrm{m} /$ and $/ \mathrm{u} /$ mouth shapes, respectively.

Leopold, Rhodes, Müller, and Jeffery (2005) found that the magnitude of face aftereffects decreased as the length of time between adaptation and test increased. Since the aftereffects observed in our experiment were driven by differences in responses in the postadaptation test phase (see planned comparisons above), we compared PSEs derived from responses in the first eight trials of the postadaptation test and those derived from the remaining seven trials. A repeated measures ANOVA with the factors lip-speech adaptation $(/ \mathrm{m} /$ or $/ \mathrm{u} /)$, adaptation identity (same or different identity as at test), and test half (first or second half) revealed a significant main effect of lipspeech adaptation $\left[F(1,15)=57.03, p<.001, \eta_{\mathrm{p}}^{2}=.79\right.$; mean PSE following adaptation to $/ \mathrm{m} /$ mouth shapes = 7.04, SEM = .22; mean PSE following adaptation to $/ \mathrm{u} /$ mouth shapes $=8.31, S E M=.20]$. There were no other significant effects (all $F_{s}<1.65$, all $p \mathrm{~s}>.21$, all $\eta_{\mathrm{p}}^{2} \mathrm{~s}<$ .10). Descriptive statistics for each combination of factors in this analysis are shown in Table 2.

The by-items analyses described above demonstrate that lip-speech aftereffects generalize across different faces. Since each of the different faces shown at test were judged by different groups of participants, these analyses also present evidence that the observed lip-speech aftereffect generalizes across groups of individuals. To investigate this issue further, we conducted a final analysis in which participants served as the unit of analysis. Because each participant judged only one face continuum at test and judged each item from that continuum only once, PSEs calculated for each observer would be extremely unreliable (i.e., they would be based on curves derived from very few observations). Thus, we used an alternative dependent variable in this final analysis.

First, we calculated the proportion of trials on which each participant judged the five most ambiguous faces on the continuum that they were shown (i.e., the physical midpoint and the two images to either side) to be saying $/ \mathrm{u} /$. Next, we analyzed these scores using a mixed-design ANOVA with the between-subjects factors lip-speech adaptation $(/ \mathrm{m} /$ or $/ \mathrm{u} /$ ) and adaptation identity (same or different identity as at test) and the within-subjects factor test phase (preadaptation or postadaptation). Because item order was fully randomized across participants, the factor test half that was included in the previous by-items analysis was not included in this by-participants analysis. The mixed-design ANOVA revealed a significant interaction between test phase and lip-speech adaptation $[F(1,408)=$ $\left.72.06, p<.001, \eta_{\mathrm{p}}^{2}=.15\right]$ and no other significant effects (all $F_{\mathrm{s}}<1.10$, all $p \mathrm{~s}>.30$, all $\left.\eta_{\mathrm{p}}^{2} \mathrm{~s}<.004\right)$. Consistent with our previous by-items analyses, paired samples $t$ tests showed that participants were less likely to label ambiguous stimuli as saying $/ \mathrm{u} /$ following adaptation to $/ \mathrm{u} /$ mouth shapes than they were in the pretest $[t(211)=-5.37, p<$ $\left..001, \eta_{\mathrm{p}}^{2}=.12\right]$, but they were more likely to label ambiguous stimuli as saying $/ \mathrm{u} /$ following adaptation to $/ \mathrm{m} / \mathrm{mouth}$ shapes than they were in the pretest $[t(199)=6.71, p<$ $\left..001, \eta_{\mathrm{p}}^{2}=.19\right]$. Descriptive statistics for each combination of factors in this analysis are shown in Table 3.

\section{DISCUSSION}

Both by-items and by-participants analyses demonstrated that participants were significantly more likely to label ambiguous faces as saying / $\mathrm{u}$ / following adaptation to $/ \mathrm{m} /$ mouth shapes than they were in the preadaptation test,

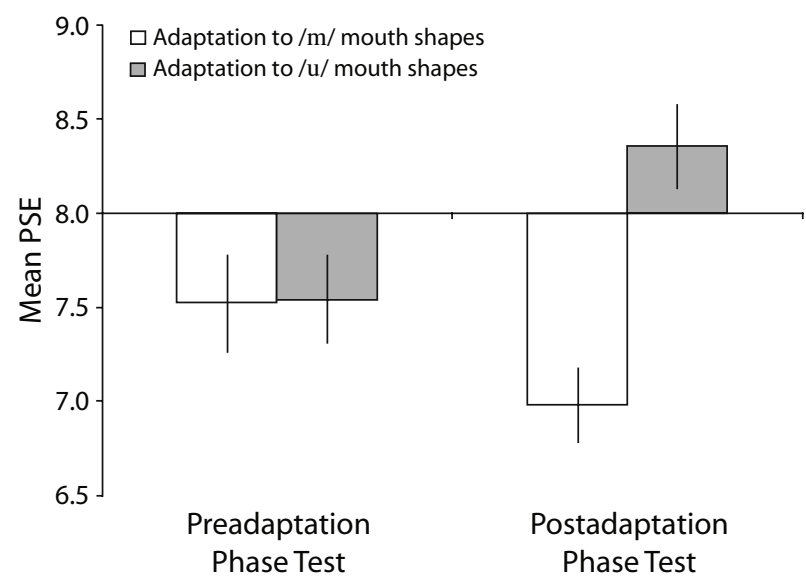

Figure 2. The significant interaction between lip-speech adaptation and test phase. Bars show mean PSE $( \pm 1 S E M)$. The $\boldsymbol{x}$-axis crosses the $\boldsymbol{y}$-axis at the physical midpoint on the $/ \mathrm{m} /$ to /u/ continua (i.e., 8). High PSE values indicate that the PSE was closer to the /u/ end of the continuum and low PSE values indicate that the PSE was closer to the $/ \mathrm{m} /$ end of the continuum. 

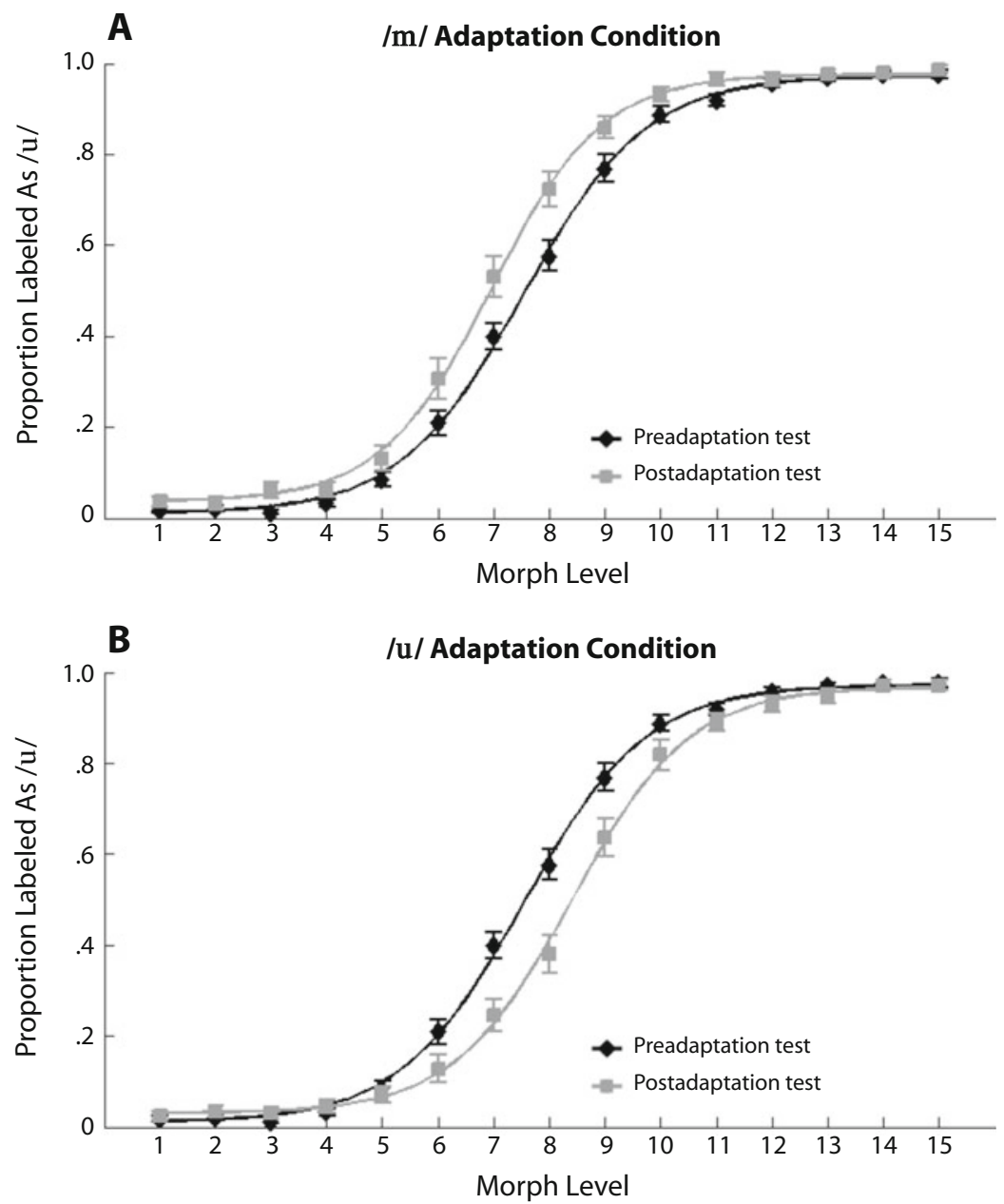

Figure 3. (A) The logistic curves fitted to data from the pre- and postadaptation phase tests for participants adapted to $/ \mathrm{m} /$ mouth shapes. (B) The logistic curves fitted to data from the pre- and postadaptation phase tests for participants adapted to $/ \mathrm{u} /$ mouth shapes. Error bars show SEM. The 1st level of morphing denotes the veridical (i.e., $100 \%$ ) $/ \mathrm{m} /$ images, and the 15 th level denotes the veridical $/ \mathrm{u} /$ images.

but they were significantly less likely to label ambiguous faces as saying $/ \mathrm{u} /$ following adaptation to $/ \mathrm{u} /$ mouth shapes than they were in the preadaptation test. Thus, our findings suggest that visual adaptation to mouth shapes associated with different sounds biases perceptions of ambiguous lip speech. The magnitude of the lip-speech aftereffect was equivalent, regardless of whether the same individual was shown in the adaptation and test phases, suggesting that the lip-speech aftereffect is not a consequence of changes to identity-specific representations of faces. Moreover, the lip-speech aftereffect occurred even though the faces shown at test and in the adaptation phase differed in size, suggesting that it is unlikely that it simply reflected low-level (i.e., retinal) aftereffects. Although several studies have demonstrated aftereffects following adaptation to natural variations in appearance (e.g., difference in sex, ethnicity, or expression; Bestelmeyer et al., 2010; Hsu \& Young, 2004; Webster et al., 2004), ours is the first to demonstrate that adaptation to mouth shapes associated with different spoken sounds can affect perceptions of ambiguous lip speech.
Previous research on auditory recalibration of lipspeech perception suggests that hearing a spoken sound biases perceptions of ambiguous lip speech, such that the ambiguous lip speech is more likely to be perceived as saying the previously presented sound (Eisner \& McQueen, 2005; van Linden \& Vroomen, 2007; Vroomen \& Baart, 2009). By contrast, our findings for visual recalibration of lip-speech perception suggest that visual adaptation to mouth shapes associated with different spoken sounds decreases the likelihood of participants interpreting ambiguous lip speech as saying that sound. Collectively, these findings for different effects of auditory and visual recalibration on perceptions of lip speech suggest that different mechanisms and processes underpin auditory and visual recalibration of lip-speech perception. Further research on the similarities and differences between auditory and visual recalibration of lip-speech perception may provide important insights into the mechanisms and processes through which different types of experience influence how we process lip speech. 
Table 2

Descriptive Statistics for Each Combination of Lip-Speech Adaptation $(/ \mathrm{m} /$ or $/ \mathrm{u} /$ ), Adaptation Identity (Same or Different Identity As at Test), and Test Half (First or Second Half) From Our Analysis of Responses in the Postadaptation Test Phase of the Experiment

\begin{tabular}{lclcl}
\hline Test Half & $\begin{array}{c}\text { Lip-Speech } \\
\text { Adaptation } \\
\text { Condition }\end{array}$ & $\begin{array}{c}\text { Adaptation } \\
\text { Identity } \\
\text { Condition }\end{array}$ & $\begin{array}{c}\text { Mean Point } \\
\text { of Subjective } \\
\text { Equality }\end{array}$ & SEM \\
\hline \multirow{2}{*}{ First } & $/ \mathrm{m} /$ & Different & 7.06 & 0.30 \\
& & Same & 6.94 & 0.26 \\
& $/ \mathrm{u} /$ & Different & 8.36 & 0.41 \\
& & Same & 8.43 & 0.24 \\
Second & $/ \mathrm{m} /$ & Different & 7.30 & 0.35 \\
& & Same & 6.87 & 0.23 \\
& & Different & 8.03 & 0.26 \\
& & Same & 8.40 & 0.27
\end{tabular}

Note-Individual test continua served as the unit of analysis.

As mentioned above, previous research on recalibration of perceptions of lip speech has emphasized the effects of recalibration following exposure to auditory stimuli (Eisner \& McQueen, 2005; van Linden \& Vroomen, 2007; Vroomen \& Baart, 2009). By contrast with this emphasis on auditory recalibration, our findings suggest that perceptions of lip speech also can be recalibrated by visual adaptation to different mouth shapes. Although our findings present novel converging evidence for plasticity in perceptions of lip speech, the function of the observed recalibration remains unclear. Given that production of successive identical visemes occurs relatively infrequently during social interaction, one possibility is that decreased sensitivity to recently seen mouth shapes in ambiguous lip speech functions to streamline processing of lip speech, decreasing the likelihood that ambiguous lip speech will be perceived as saying the same sound as that associated with immediately preceding visemes. That the duration of the adaptation period in our study (48 sec) would also be extremely rare during normal social interaction represents an important caveat to the conclusions that can be drawn in this regard, however. On this point, we note here that previous research has shown that, although the magnitude of face aftereffects is related positively to the duration of exposure (Leopold et al., 2005), robust face aftereffects have been observed following relatively brief exposure (i.e., 1,000 msec) to the adapting stimuli (Leopold et al., 2005). Thus, we do not necessarily discount the possibility that the type of face aftereffects that we observed in the present study may influence lip-speech perception during normal social interaction, albeit at a reduced magnitude of perceptual effect. Further research is needed to investigate whether lip-speech aftereffects occur following adaptation periods that more closely reflect the duration of visemes that are produced during typical speech and, if lip-speech aftereffects do occur under such circumstances, what are the temporal dynamics of their decay. Although Leopold et al. (2005) found that face aftereffects decayed fairly rapidly, responses in the first and second half of our postadaptation test did not differ significantly, suggesting that lip-speech aftereffects may not decay as quickly as some other types of face aftereffects appear to do.

Although it is unclear whether adaptation to transient mouth shapes that may be seen during social interactions influences perceptions of lip speech, our findings for lipspeech aftereffects following relatively prolonged exposure to different mouth shapes may still play an important role in lip-speech perception during normal social interactions. There is considerable shape variation among static faces (i.e., faces making no emotional expression or that are not speaking). Consequently, a challenge for the perceptual system is distinguishing idiosyncratic variations in resting face shape (i.e., variations that are unrelated to speech or emotional expression, for example) from dynamic variations in face shape that are related to speech, emotion, or other variant social signals. Decreased sensitivity to adapted mouth shapes in ambiguous stimuli may, therefore, function to reduce the likelihood of variations in resting mouth shape that are unrelated to speech influencing perceptions of lip speech. For example, when one interacts with an individual with unusually thin lips, adaptation to their natural resting mouth shape may reduce the likelihood of ambiguous lip speech that they may produce during the interaction being perceived as a cue that they are saying $/ \mathrm{m} /$. Similarly, when one interacts with an individual who has "pouted" (or pursed) lips, adaptation to their natural resting mouth shape may reduce the likelihood of ambiguous lip speech that they may produce during the interaction being perceived as a cue that they are saying /u/. Thus, the lip-speech aftereffects observed in the present study may function to reduce the effects of "noise" in the perception of lip speech during interactions with individuals whose natural resting mouth shape could otherwise be mistaken for lip speech.

Our findings demonstrate a recalibration of perception of facial cues associated with spoken sounds following visual adaptation to mouth shapes, and they present novel converging evidence that adaptation to natural variations in appearance influences face perception. More importantly, our findings, together with recent studies showing effects of adaptation to gaze direction (Calder et al., 2008; Jenkins et al., 2006), emotional expressions (e.g., Bestelmeyer et al., 2010; Hsu \& Young, 2004; Webster

Table 3

Descriptive Statistics for Each Combination of Lip-Speech Adaptation ( $/ \mathrm{m} /$ or $/ \mathrm{u} /$ ), Adaptation Identity (Same or Different Identity As at Test), and Test Phase (Pre- or Postadaptation Test) in Our By-Subjects Analysis

\begin{tabular}{ccccc}
\hline $\begin{array}{c}\text { Lip-Speech } \\
\text { Adaptation } \\
\text { Condition }\end{array}$ & $\begin{array}{c}\text { Adaptation } \\
\text { Identity } \\
\text { Condition }\end{array}$ & Test Phase & $\begin{array}{c}\text { Mean Proportion } \\
\text { of Ambiguous } \\
\text { Faces Labeled /u/ }\end{array}$ & SEM \\
\hline \multirow{2}{*}{$/ \mathrm{m} /$} & Different & $\begin{array}{l}\text { Preadaptation } \\
\text { Postadaptation }\end{array}$ & .57 & .03 \\
& \multirow{2}{*}{ Same } & Preadaptation & .58 & .03 \\
& & Postadaptation & .67 & .03 \\
& \multirow{2}{*}{ Different } & Preadaptation & .55 & .03 \\
& & Postadaptation & .45 & .03 \\
& \multirow{2}{*}{ Same } & Preadaptation & .57 & .03 \\
& & Postadaptation & .43 & .03 \\
\hline
\end{tabular}


et al., 2004), and head orientation (e.g., Fang et al., 2007), present further evidence that adaptation to variant facial cues that can change rapidly within a given individual can affect face perception. Although the present study demonstrates that visual adaptation to mouth shapes associated with spoken sounds can bias perceptions of ambiguous lip speech, further research exploring how such aftereffects influence the integration of facial and auditory cues may shed further light on the role of face aftereffects in the processing of speech.

\section{AUTHOR NOTE}

Address correspondence to B. C. Jones, School of Psychology, University of Aberdeen, Aberdeen, Scotland (e-mail: ben.jones@abdn.ac.uk).

\section{REFERENCES}

Bertelson, P., Vroomen, J., \& de Gelder, B. (2003). Visual recalibration of auditory speech identification: A McGurk aftereffect. Psychological Science, 14, 592-597.

Bestelmeyer, P. E. G., Jones, B. C., DeBruine, L. M., Little, A. C., Perrett, D. I., Schneider, A., ET AL. (2008). Sex-contingent face aftereffects depend on perceptual category rather than structural encoding. Cognition, 107, 353-365. doi:10.1016/j.cognition.2007.07.018

Bestelmeyer, P. E. G., Jones, B. C., DeBruine, L. M., Little, A. C., Schneider, A., Welling, L. L. M., \& Conway, C. A. (2010). Face aftereffects demonstrate interdependent processing of expressions and the invariant characteristics of sex and race. Visual Cognition, 18, 255-274.

Bruce, V., \& Young, A. W. (1986). Understanding face recognition. British Journal of Psychology, 77, 305-327.

Calder, A. J., Jenkins, R., Cassel, A., \& Clifford, C. W. G. (2008). Visual representation of eye gaze is coded by a nonopponent multichannel system. Journal of Experimental Psychology: General, 137, 244-261. doi:10.1037/0096-3445.137.2.244

DeBruine, L. M., Jones, B. C., Unger, L., Little, A. C., \& Feinberg, D. R. (2007). Dissociating averageness and attractiveness: Attractive faces are not always average. Journal of Experimental Psychology: Human Perception \& Performance, 33, 1420-1430.

EISNER, F., \& McQueEn, J. M. (2005). The specificity of perceptual learning in speech processing. Perception \& Psychophysics, 67, 224238.

FANG, F., IJICHI, K., \& He, S. (2007). Transfer of the face viewpoint aftereffect from adaptation to different and inverted faces. Journal of Vision, 7(13, Art. 6), 1-9.

García-Pérez, M. A., \& Alcalá-Quintana, R. (2005). Sampling plans for fitting the psychometric function. Spanish Journal of Psychology, 8, 256-289.

Haxby, J. V., Hoffman, E. A., \& Gobbini, M. I. (2000). The distributed human neural system for face perception. Trends in Cognitive Sciences, 4, 223-233

Hsu, S.-M., \& Young, A. W. (2004). Adaptation effects in facial expression recognition. Visual Cognition, 11, 871-899.

JaQUet, E., Rhodes, G., \& HaYward, W. G. (2007). Opposite aftereffects for Chinese and Caucasian faces are selective for social category information and not just physical face differences. Quarterly Journal of Experimental Psychology, 60, 1457-1467.
JefFery, L., Rhodes, G., \& Busey, T. (2006). View-specific coding of face shape. Psychological Science, 17, 501-505. doi:10.1111/j.1467 $-9280.2006 .01735 . x$

Jenkins, R., Beaver, J. D., \& CALDER, A. J. (2006). I thought you were looking at me: Direction-specific aftereffects in gaze perception. Psychological Science, 17, 506-513.

Jones, B. C., DeBruine, L. M., \& Little, A. C. (2008). Adaptation reinforces preferences for correlates of attractive facial cues. Visual Cognition, 16, 849-858. doi:10.1080/13506280701760811

Leopold, D. A., O'Toole, A. J., Vetter, T., \& Blanz, V. (2001). Prototype-referenced shape encoding revealed by high-level aftereffects. Nature Neuroscience, 4, 89-94.

Leopold, D. A., Rhodes, G., Müller, K.-M., \& JefFery, L. (2005). The dynamics of visual adaptation to faces. Proceedings of the Royal Society B, 272, 897-904.

Little, A. C., DeBruine, L. M., \& Jones, B. C. (2005). Sex-contingent face aftereffects suggest distinct neural populations code male and female faces. Proceedings of the Royal Society B, 272, 1-5. doi:10.1098/ rspb.2005.3220

Little, A. C., DeBruine, L. M., Jones, B. C., \& Waitt, C. (2008). Category contingent aftereffects for faces of different races, ages and species. Cognition, 106, 1537-1547.

MCGURK, H., \& MACDonALD, J. (1976). Hearing lips and seeing voices. Nature, 264, 746-748.

Rhodes, G., Jeffery, L., Watson, T. L., Clifford, C. W. G., \& NaKAYAMA, K. (2003). Fitting the mind to the world: Face adaptation and attractiveness aftereffects. Psychological Science, 14, 558-566.

Rotshtein, P., Henson, R. N. A., Treves, A., Driver, J., \& Dolan, R. J. (2005). Morphing Marilyn into Maggie dissociates physical and identity face representations in the brain. Nature Neuroscience, $\mathbf{8}$, 107-113. doi:10.1038/nn1370

Tiddeman, B. P., Perrett, D. I., \& Burt, D. M. (2001). Prototyping and transforming facial textures for perception research. IEEE Computer Graphics \& Applications, 21, 42-50.

van Linden, S., \& VRoOmen, J. (2007). Recalibration of phonetic categories by lipread speech versus lexical information. Journal of Experimental Psychology: Human Perception \& Performance, 33, 1483-1494.

VROOMEN, J., \& BAART, M. (2009). Recalibration of phonetic categories by lipread speech: Measuring aftereffects after a 24-hour delay. Language \& Speech, 52, 341-350.

Webster, M. A., Kaping, D., Mizokami, Y., \& Duhamel, P. (2004). Adaptation to natural facial categories. Nature, 428, 557-561.

Webster, M. A., \& MacLin, O. H. (1999). Figural aftereffects in the perception of faces. Psychonomic Bulletin \& Review, 6, 647-653.

Winston, J. S., Henson, R. N. A., Fine-Goulden, M. R., \& Dolan, R. J. (2004). fMRI-adaptation reveals dissociable neural representations of identity and expression in face perception. Journal of Neurophysiology, 92, 1830-1839.

Xu, H., Dayan, P., LiPkin, R. M., \& Qian, N. (2008). Adaptation across the cortical hierarchy: Low-level curve adaptation affects high-level facial-expression judgments. Journal of Neuroscience, 28, 3374-3383. doi:10.1523/JNEUROSCI.0182-08.2008

Yamashita, J. A., Hardy, J. L., De Valois, K. K., \& Webster, M. A. (2005). Stimulus selectivity of figural aftereffects for faces. Journal of Experimental Psychology: Human Perception \& Performance, 31, 420-437. doi:10.1037/0096-1523.31.3.420

(Manuscript received July 8, 2009; revision accepted for publication February 2, 2010.) 